\title{
Sol-gel transition study and pyrolysis of alumina-based gels prepared from alumatrane precursor
}

\author{
Bussarin Ksapabutr ${ }^{\mathrm{a}}$, Erdogan Gulari ${ }^{\mathrm{b}}$, Sujitra Wongkasemjit ${ }^{\mathrm{a}, *}$ \\ ${ }^{a}$ The Petroleum and Petrochemical College, Chulalongkorn University, Bangkok 10330, Thailand \\ ${ }^{\mathrm{b}}$ Department of Chemical Engineering, University of Michigan, Ann Arbor, MI 48109-2136, USA
}

Received 25 May 2003; accepted 18 November 2003

\begin{abstract}
Alumina gels were prepared by the sol-gel method using alumatrane or tris(alumatranyloxy-i-propyl)amine as precursor synthesised directly from the reaction of inexpensive and readily available compounds, aluminium hydroxide and triisopropanolamine (TIS), via the one step process. Sol-gel process parameters, such as gel time, were correlated to variables of the initial stage of the process, such as $\mathrm{pH}$, temperature of hydrolysis and hydrolysis ratio. The sol-gel transition of alcoholic alumatrane solutions was monitored by multiple waveform rheological measurements. The gel time could be determined from the evolution of the storage $\left(G^{\prime}\right)$ and loss $\left(G^{\prime \prime}\right)$ moduli versus time at different frequencies using the Winter Chambon criterion (convergence of the loss tangents at the gel point). Increasing $\mathrm{pH}$ values, hydrolysis ratio and/or temperature accelerated the kinetics of hydrolysis-condensation reactions and thus reduced the gel time. The apparent activation energy of the cross-linking leading to the gelation calculated from the gel times at various temperatures was found to be approximately $139 \mathrm{~kJ} \mathrm{~mol}^{-1}$ and independent of hydrolysis ratio. Alumina materials prepared from the heat treatment of obtained gel at $500{ }^{\circ} \mathrm{C}$ were analysed using X-ray diffraction and the BET method.
\end{abstract}

(C) 2003 Elsevier B.V. All rights reserved.

Keywords: Sol-gel transition; Alumatrane precursor; Alumina; Rheology; Gel time

\section{Introduction}

Alumina materials have a wide range of applications in a great number of industrial areas, particularly in catalysis, membrane separation processes, catalytic membrane reactors, adsorbent, composite, coating, fibre, electronic and optic fields [1-7]. Alumina prepared by sol-gel process is frequently used in such areas. In the development of soft chemistry, the sol-gel process is considered to be the most practical method for the synthesis of inorganic oxides. This process usually involves the hydrolysis and condensation of various metal alkoxide molecules under controlled conditions to form metal-oxygen-metal bridging units. In many cases the metal alkoxide precursors are very sensitive to water and, therefore, cannot control the hydrolysis reaction $[8,9]$. Generally, the aluminium alkoxides, such as aluminium sec-butoxide and aluminium

* Corresponding author. Tel.: +66-2-218-4133; fax: +66-2-215-4459.

E-mail address: dsujitra@chula.ac.th (S. Wongkasemjit). iso-propoxide, are used to prepare alumina by sol-gel method [10-12]. However, these usual precursors are expensive and aluminium $s e c$-butoxide can be rapidly hydrolysed to give the hydrolysis products dispersed in 2-butanol [13-17].

Alumatrane is aminoalkoxide derivatives of aluminium synthesised directly from the reaction of inexpensive and readily available compounds, aluminium hydroxide and triisopropanolamine (TIS), via the one step process. Its presence of trialkanoamine ligands is hydrolytically stable, thus yielding more controllable chemistry and minimising special handling requirement. Therefore, it should be used as precursor in ceramic processing by sol-gel route. In this work, we investigate the dynamic viscoelastic properties and FT-IR studies on sol-gel process of alumatrane under different conditions. This observation is mainly focused on the influence of $\mathrm{pH}$, hydrolysis ratio and temperature on the cross-linking process. In addition, some preliminary thermal studies of an alumina obtained by the sol-gel technique are also observed. 


\section{Experimental}

\subsection{Materials}

The reagents and preparation of alumatrane were those used in Wongkasemjit's work [18]. Aluminium hydroxide hydrate $\left(\mathrm{Al}(\mathrm{OH})_{3}\right)$ was purchased from Sigma Chemical Co. and TIS was obtained from Fluka Chemical Co. Both were used as received. Ethylene glycol (EG), used as solvent in the reaction, was purchased from Farmitalia Carlo Erba and purified by fractional distillation at $200^{\circ} \mathrm{C}$, under $\mathrm{N}_{2}$ before use. Triethylenetetramine (TETA) was purchased from Facai Polytech. Co. Ltd. and distilled under vacuum $(0.1 \mathrm{mmHg})$ at $130^{\circ} \mathrm{C}$ prior to use. Methanol and acetonitrile were purchased from Lab-Scan Company Co. Ltd. and purified by standard techniques. Methanol was distilled over magnesium activated with iodine. Acetonitrile was distilled over calcium hydride powder. Nitric acid and ammonia solution used as catalysts in sol-gel processing were purchased from Lab-Scan Company Co. Ltd. and Merck Co., respectively.

The synthesis of alumatrane was carried out in one step. A mixture of $\mathrm{Al}(\mathrm{OH})_{3}$, TIS and TETA was suspended in EG, and heated to the boiling point of EG under nitrogen to distill off ethylene glycol along with removal of water liberated from the reaction. The solution was virtually clear, indicating reaction completion. The reaction mixture was cooled at room temperature. The product precipitated out as a white solid. The solid was separated, washed with acetonitrile and dried under vacuum $(0.1 \mathrm{mmHg})$ at room temperature.

\subsection{Preparation and heat treatment of alumina gels}

Alumatrane or tris(alumatranyloxy-i-propyl)amine was used as an alumina source or the precursor of the sol-gel derived system under at various $\mathrm{pH}$ values, temperature and hydrolysis ratio. The preparation of alumatrane has already been reported in a previous paper [18]. Alumatrane was dissolved in dried methanol for a period. This methanolic alumatrane solution was checked using FT-IR spectroscopy and TGA to confirm the absence of alcohol interchange reactions. Then water was added together with catalyst, except the case of $\mathrm{pH} 9$ where no catalyst was added. For acid conditions at $\mathrm{pH} 3$ and $5, \mathrm{HNO}_{3}$ was used, and for neutral condition at $\mathrm{pH} 7, \mathrm{HNO}_{3}$ was also used to adjust $\mathrm{pH}$ value of alcoholic alumatrane solution having $\mathrm{pH}$ 9. To attain basic conditions, $\mathrm{pH} 10$ and $11, \mathrm{NH}_{4} \mathrm{OH}$ was added. Three hydrolysis ratios $\left(h=\left[\mathrm{H}_{2} \mathrm{O}\right] /[\mathrm{Al}]\right)$ of 9,18 and 27 and six $\mathrm{pH}$ values ranging from 3 to 11 were investigated due to gellability zone while the mole ratio of methanol to [Al] was kept at 60 . The solution was mixed with a vigorous stirring at the temperature range varied from 20 to $36^{\circ} \mathrm{C}$.

Alumina powders were produced by heat treatment of the resulting gels at various $\mathrm{pH}$ values in a furnace at $500{ }^{\circ} \mathrm{C}$ and held at the final temperature for $7 \mathrm{~h}$.

\subsection{Measurements}

\subsubsection{Sol-gel process}

2.3.1.1. Rheological characterisations. The rheological measurements, which were performed to determine sol-gel transition and dynamic viscoelastic properties, were carried out using a Rheometric Scientific Inc. (Model ARES) with a cone-plate configuration covered with the humidity chamber. The humidity chamber filled with methanol to obtain a saturated atmosphere was used to prevent evaporation of solvent during measurement. The dimensions corresponding to the geometry were $25 \mathrm{~mm}$ for the diameter and $0.04^{\circ}$ for the angle. The minimum distance between plate and truncated cone was $0.051 \mathrm{~mm}$. The temperature control was achieved with a thermostated-circulating bath. The sample was stirred until homogeneous and loaded in the rheometer. The time for loading the sample was kept to a minimum so as to reduce the solvent evaporation. The experimental conditions (applied strain amplitude and frequency sweep range) were fixed after preliminary experiments. The amplitude was fixed within the linear viscoelastic range. The multiwave experiments were run with eight frequencies ranging from 0.2 to $1.6 \mathrm{rad} \mathrm{s}^{-1}$ and the strains $(\gamma)$ were kept at $3 \%$ at each harmonic. A narrow frequency range was employed because their properties change markedly with time. In addition, no intersection of the $\tan \delta$ curves at a certain point identified as gel point was observed at higher frequencies.

Although the time requested for $G^{\prime}$ and $G^{\prime \prime}$ to be equal is designated as the gel point by some authors $[19,20]$, a more accurate investigation of the gel point has been given by Winter and co-workers as the point where $\tan \delta\left(=G^{\prime \prime} / G^{\prime}\right)$ is independent of frequency. The gel point can be precisely identified and located by the study of the evolution of $G^{\prime}$ and $G^{\prime \prime}$ as a function of the frequency at various reaction times near the gel point. This criterion was firstly proposed for chemical gels, but has also been applied to physical gels [21-23]. For such systems the linear viscoelastic behaviour at gel point is described by the gel equation $[21,22]$.

$\tau(t)=S \int_{-\infty}^{t}\left(t-t^{\prime}\right)^{-n} \dot{\gamma}\left(t^{\prime}\right) \mathrm{d} t^{\prime}, \quad 0<n<1$

where $\tau$ is the stress tensor, $\dot{\gamma}$ is the rate of deformation tensor, $S$ is the strength of the network at the gel point depending on the flexibility of molecular chains and cross-links, and on the cross-link density. The value of relaxation exponent, $n$, varies over about the entire possible range between 0 and 1 indicating a strongly elastic gel $(n=0)$ or a more viscous gel $(n=1)$ [25]. The dynamic mechanical behaviour at the gel point is given by a power law relation between moduli $\left(G^{\prime}\right.$ and $\left.G^{\prime \prime}\right)$ and the angular frequency $(\omega)$

$G^{\prime}(\omega) \sim G^{\prime \prime}(\omega) \sim \omega^{n}$

i.e. storage $\left(G^{\prime}\right)$ and loss moduli $\left(G^{\prime \prime}\right)$ are parallel in a $\log -\log$ plot. Consequently, the loss angle $(\delta)$ is independent 
of frequency at the gel point but proportional to the relaxation exponent:

$\delta_{\mathrm{c}}=\frac{n \pi}{2} \quad$ or $\quad \tan \delta_{\mathrm{c}}=\frac{G^{\prime \prime}}{G^{\prime}}=\tan \left(\frac{n \pi}{2}\right)$

Therefore, the gel point can be conveniently determined using the evolution of $\tan \delta$ at different frequencies as a function of reaction time. The various curves intersect at one critical point, at which $\tan \delta$ becomes independent of frequency. The time at which this occurs is the gel time [24,25].

In very recent years, some studies have also been used a modification of self-similarity method to determine the gel time. It is useful for systems exhibiting no intersection of the curves, but is also applicable to systems whose curves coincide at a specific point. The gel point is associated with the occurrence of statistical loss factor self-similarity. In this way, the gel time is obtained by the minimum of the curve $\log (s /(\tan \delta))$ as a function of reaction time where $s$ is the standard deviation of $\tan \delta$ in a frequency sweep [26].

2.3.1.2. FT-IR spectroscopic characterisations. The hydrolysis and condensation of alumatrane as a function of the reaction time were followed using FT-IR spectroscopy. An FT-IR spectrophotometer (Nicolet, NEXUS 670) with 16 scans at a resolution of $4 \mathrm{~cm}^{-1}$ was used in this study.

\subsubsection{Characterisation of pyrolysed gels}

Alumina supports obtained from annealing the gel at $500{ }^{\circ} \mathrm{C}$ were characterised using BET method and X-ray diffraction techniques. BET surface area and pore size distribution were measured by using nitrogen at $77 \mathrm{~K}$ in Autosorb-1 gas sorption system (Quantasorb JR.). Samples were degassed at $200^{\circ} \mathrm{C}$ under a reduced pressure prior to each measurement. The structure of the phases in the samples annealed at various temperatures was obtained at room temperature using X-ray powder diffraction. XRD spectra were recorded on D/MAX 2000 series (Rigaku Co.) using $\mathrm{Cu} \mathrm{K} \alpha$ radiation $(\lambda=0.154 \mathrm{~nm})$.

\section{Results and discussion}

\subsection{Sol-gel process}

\subsubsection{Gel times and dynamic viscoelastic properties by rheological measurements}

The evolution of gelation process with time was investigated in eight different frequencies ranging from 0.2 to $1.6 \mathrm{rad} \mathrm{s}^{-1}$. Fig. 1a and $\mathrm{b}$ show measured values of storage $G^{\prime}$ and loss $G^{\prime \prime}$ moduli, respectively, as a function of reaction time for the system with $\mathrm{pH} 7$ and $h=9$ at $25^{\circ} \mathrm{C}$. In the initial part of the reaction, $G^{\prime \prime}$ is greater than $G^{\prime}$, which is a characteristic feature of liquids. Furthermore, this result can be observed clearer in Fig. 4 which illustrates variations in $G^{\prime}$ and $G^{\prime \prime}$ with applied frequencies at different reaction times. With continuing gelation reaction, both the moduli
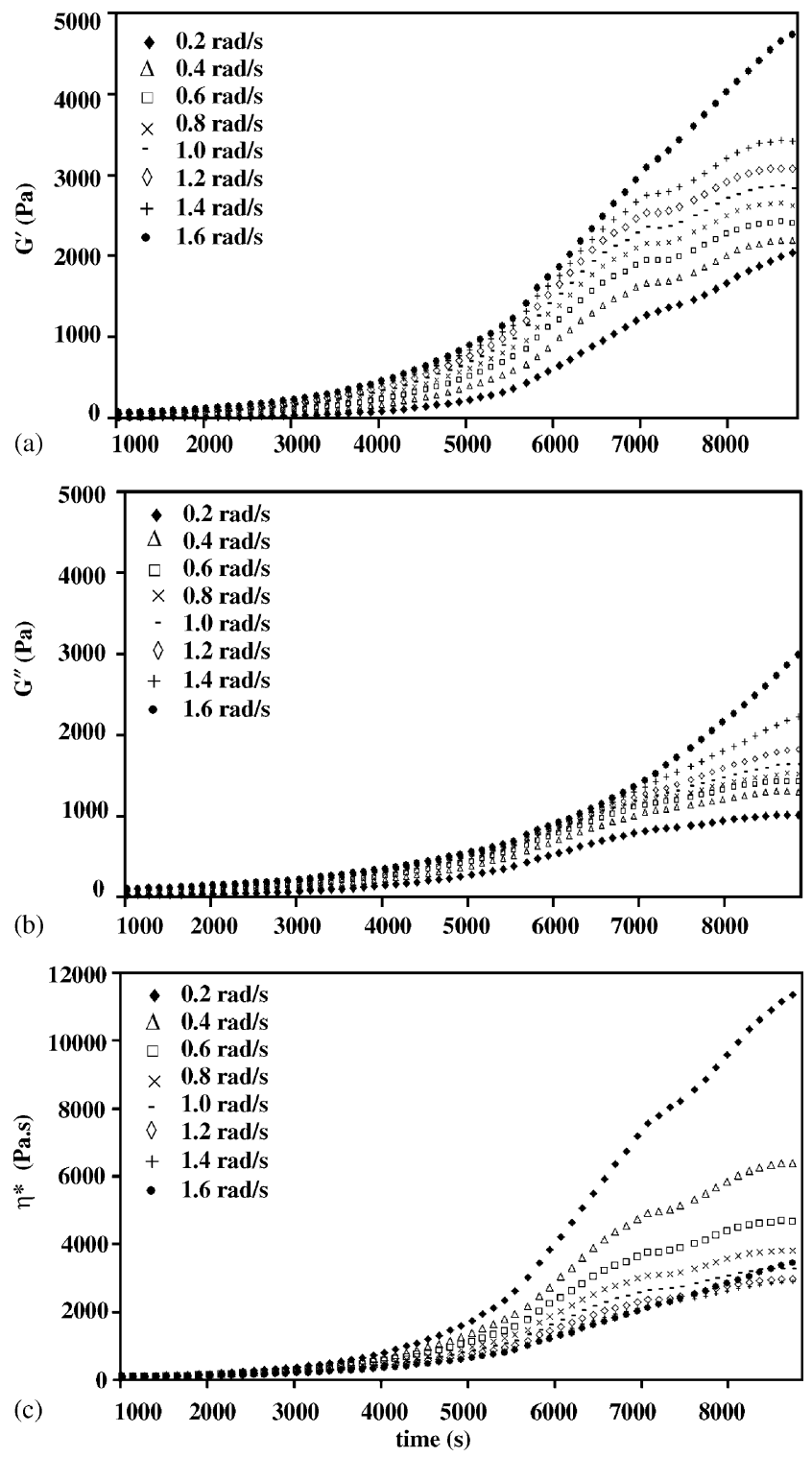

Fig. 1. Plots showing (a) storage modulus $\left(G^{\prime}\right)$; (b) loss modulus $\left(G^{\prime \prime}\right)$ and (c) complex viscosity $\left(\eta^{*}\right)$ as a function of the reaction time for the system $\mathrm{pH} 7$ and $h=9$ at $25^{\circ} \mathrm{C}$.

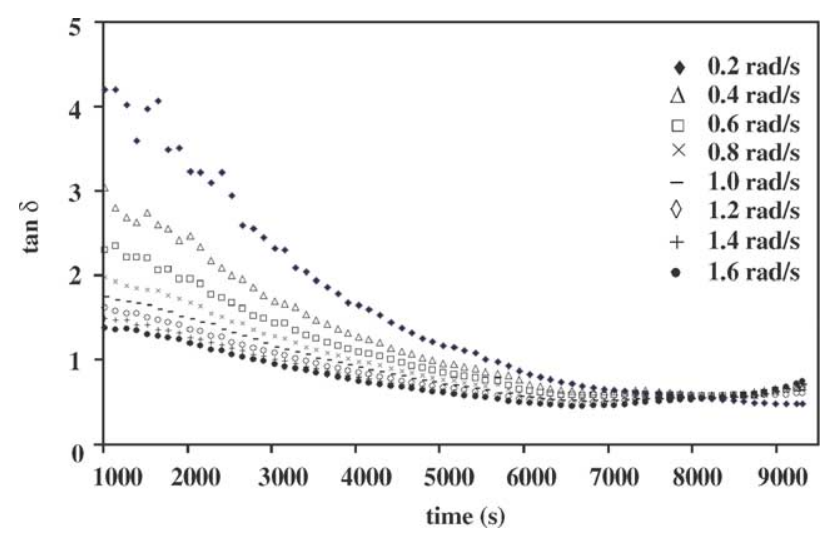

Fig. 2. Variation of $\tan \delta$ during sol-gel processing as a function of the time for $\mathrm{pH} 7$ at $h=9$ and temperature of $25^{\circ} \mathrm{C}$. 


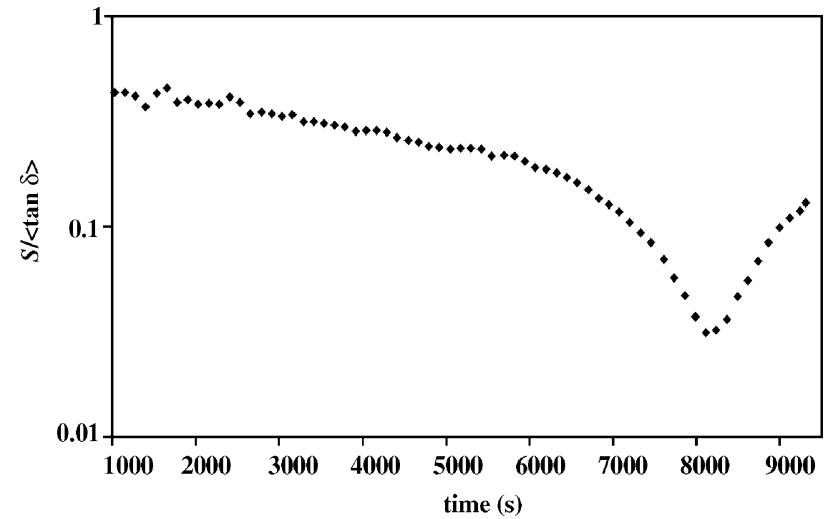

Fig. 3. Determination of the gel time using the statistical loss factor self-similarity method for $\mathrm{pH} 7$ at $h=9$ and temperature of $25^{\circ} \mathrm{C}$.

increased with time. However, $G^{\prime}$ rises more sharply than $G^{\prime \prime}$ indicating a gradual formation of gel network with a predominantly elastic character. In Fig. 1c the complex dynamic viscosity $\left(\eta^{*}\right)$ was plotted as a function of time at different frequencies. The viscosity increased as the gelation grew because more work must be done to produce flow of the sol. In addition, the complex dynamic viscosity $\eta^{*}$ increased as frequency decreased. These observations are more significant when reaction time increased indicating an evolution from liquid to solid-like state.

According to Eq. (3) the critical gel is identified by a loss $\operatorname{tangent,} \tan \delta=G^{\prime \prime} / G^{\prime}$, which is independent of frequency. Therefore, $\tan \delta$ was investigated as a function of time at different frequencies. For the system with $\mathrm{pH} 7$ and $h=9$ at $25^{\circ} \mathrm{C}$, the various curves intersected at one point, at which $\tan \delta$ became independent of frequency (Fig. 2). The time at which this occurs is the gel time $\left(t_{\text {gel }} \sim 8100 \mathrm{~s}\right)$, as depicted in Section 2.2. Knowledge of the evolution in rheological properties and gel times during sol-gel process is a useful guide for the fabrication of ceramic materials in a wide variety of forms (thin film coatings, ceramic fibres or mem-

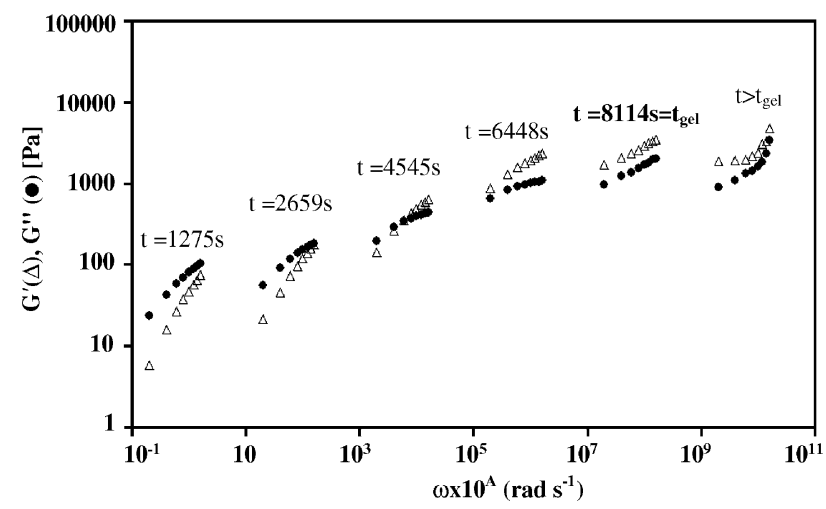

Fig. 4. Dynamic storage $\left(G^{\prime}\right)$ and loss $\left(G^{\prime \prime}\right)$ moduli as a function of the frequency $(\omega)$ for the system $\mathrm{pH} 7$ and $h=9$ at $25^{\circ} \mathrm{C}$. The curves were horizontally shifted by the factor: $A=2$ for $t=2659 \mathrm{~s} ; A=4$ for $t=4545 \mathrm{~s} ; A=6$ for $t=6448 \mathrm{~s} ; A=8$ for $t=8114 \mathrm{~s}$; and $A=10$ for $t>t_{\mathrm{gel}}$.

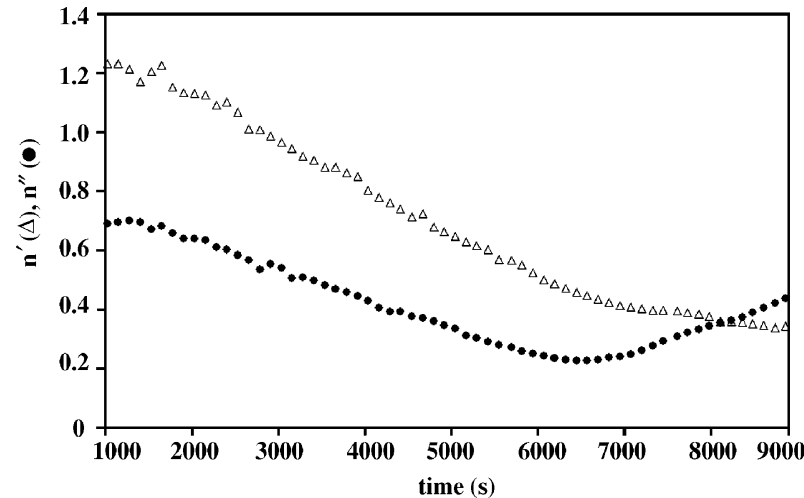

Fig. 5. Changes of viscoelastic exponent for $n^{\prime}$ and $n^{\prime \prime}$, the storage and the loss shear moduli, during the gelation reaction, obtained from fits as those shown in Fig. 4.

Table 1

$\mathrm{pH}$ effect on the gel time, and dynamic properties for $h=9$ at $25^{\circ} \mathrm{C}$

\begin{tabular}{rrrrrrl}
\hline $\mathrm{pH}$ & $t_{\text {gel }}(\mathrm{s})$ & $G^{\prime}(\mathrm{Pa})$ & $G^{\prime \prime}(\mathrm{Pa})$ & $\eta^{*}(\mathrm{Pas})$ & $G^{\prime} / G^{\prime \prime}$ & $n$ \\
\hline 3 & 16900 & 4800.45 & 1105.54 & 6157.63 & 4.34 & 0.14 \\
5 & 13100 & 2875.11 & 1293.79 & 3941.00 & 2.22 & 0.27 \\
7 & 8100 & 2556.35 & 1424.30 & 3657.94 & 1.79 & 0.33 \\
9 & 17800 & 5802.23 & 1200.03 & 7406.28 & 4.83 & 0.13 \\
10 & 5550 & 1500.92 & 989.11 & 2246.91 & 1.52 & 0.37 \\
11 & 3080 & 1003.24 & 750.18 & 1565.88 & 1.34 & 0.41 \\
\hline
\end{tabular}

branes) and to optimise the physical properties required in the final products. It is known that these gels show the property of being fluid after preparation allowing their ease of fabrication into various shapes and reach high viscosity after the gel time. From the experimental results, we also found that before the gel point, $\tan \delta$ decreased with increasing frequency, which is typical for a viscoelastic liquid. After the gel point, $\tan \delta$ increased with frequency, as the characteristic of a viscoelastic solid. Apparently, the sample had changed from a viscoelastic liquid to a viscoelastic solid. Equivalently, the gel time can be calculated by the method of statistical loss factor self-similarity, as noted above by observation of the minimum of the curve $\log (s /(\tan \delta))$ against

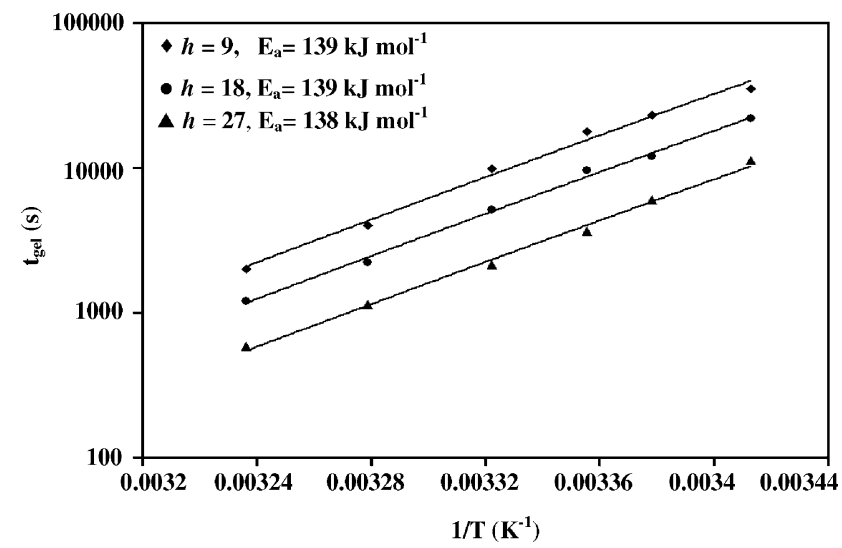

Fig. 6. Semi-logarithmic plot of gel time vs. $1 / T\left(\mathrm{~K}^{-1}\right)$. 
Table 2

Hydrolysis ratio effect on the gel time, and dynamic properties for $\mathrm{pH} 9$ at $25^{\circ} \mathrm{C}$

\begin{tabular}{rrrrlll}
\hline$h$ & $t_{\text {gel }}(\mathrm{s})$ & $G^{\prime}(\mathrm{Pa})$ & $G^{\prime \prime}(\mathrm{Pa})$ & $\eta^{*}(\mathrm{~Pa} \mathrm{~s})$ & $G^{\prime} / G^{\prime \prime}$ & $n$ \\
\hline 9 & 17800 & 5802.23 & 1200.03 & 7406.28 & 4.83 & 0.13 \\
18 & 9580 & 3003.25 & 1351.46 & 4116.65 & 2.22 & 0.27 \\
27 & 3640 & 2116.89 & 1206.62 & 3045.79 & 1.75 & 0.33 \\
\hline
\end{tabular}

reaction time. Fig. 3 illustrates that the time at the minimum point compared very well with the experimental value observed for the frequency independence of loss tangent.

The frequency dependence of $G^{\prime}$ and $G^{\prime \prime}$ for several measurement times with $\mathrm{pH} 7$ and $h=9$ at $25^{\circ} \mathrm{C}$ was exhibited in Fig. 4. At early stage of the reaction $G^{\prime}$ is smaller than $G^{\prime \prime}$. As gelation proceeded at the instant, the traces of $G^{\prime}$ and $G^{\prime \prime}$ became parallel to each other showing power law behaviour. Such a condition corresponds to a sol-gel transition according to the criterion suggested by Winter and Chambon. As noted in Section 2.2, values of $\mathrm{n}$ near the gelation critical point can be identified from power law character. The slopes of these lines gave $n$, the viscoelastic exponent in Eq. (2), for the frequency dependence of the moduli. The changes of $\mathrm{n}$ with extent of reaction were shown in Fig. 5 where $n^{\prime}$ and $n^{\prime \prime}$ for storage and loss moduli, respectively, were plotted against time. Both values decreased and then merged to 0.36 at $8114 \mathrm{~s}$. These values of $\mathrm{n}$ component were in good agreement with the values obtained from Fig. 2 and Eq. (3) At the gel point, $\tan \delta$ is equal to 0.56 , which corresponds to a relaxation exponent of $n=0.33$.
Table 3

Temperature effect on the gel time, and dynamic properties for $\mathrm{pH} 9$ and $h=9,18$ and 27

\begin{tabular}{lrrrrrl}
\hline$h$ & $t_{\text {gel }}(\mathrm{s})$ & $G^{\prime}(\mathrm{Pa})$ & $G^{\prime \prime}(\mathrm{Pa})$ & $\eta^{*}(\mathrm{Pas})$ & $G^{\prime} / G^{\prime \prime}$ & $n$ \\
\hline At $20^{\circ} \mathrm{C}$ & & & & & & \\
9 & 35000 & 7645.23 & 1287.78 & 9691.16 & 5.94 & 0.11 \\
18 & 21880 & 5134.42 & 2053.77 & 6912.43 & 2.50 & 0.24 \\
27 & 11270 & 4211.56 & 2190.56 & 5933.99 & 1.92 & 0.31 \\
At $23{ }^{\circ} \mathrm{C}$ & & & & & & \\
9 & 23140 & 6178.21 & 1173.85 & 7860.92 & 5.26 & 0.12 \\
18 & 12000 & 4214.25 & 1727.84 & 5693.38 & 2.44 & 0.25 \\
27 & 6000 & 3165.51 & 1709.37 & 4496.94 & 1.85 & 0.32 \\
At $25{ }^{\circ} \mathrm{C}$ & & & & & & \\
9 & 17800 & 5802.23 & 1200.03 & 7406.28 & 4.83 & 0.13 \\
18 & 9580 & 3003.25 & 1351.46 & 4116.65 & 2.22 & 0.27 \\
27 & 3640 & 2116.89 & 1206.62 & 3045.79 & 1.75 & 0.33 \\
At $28{ }^{\circ} \mathrm{C}$ & & & & & & \\
9 & 9900 & 5620.17 & 1348.8 & 7224.69 & 4.17 & 0.15 \\
18 & 5160 & 2534.43 & 1241.87 & 3527.92 & 2.04 & 0.29 \\
27 & 2130 & 1676.51 & 1022.67 & 2454.76 & 1.64 & 0.35 \\
At 32 ${ }^{\circ} \mathrm{C}$ & & & & & & \\
9 & 4000 & 4802.47 & 1392.72 & 6250.42 & 3.45 & 0.18 \\
18 & 2230 & 2138.73 & 1176.3 & 3051.09 & 1.82 & 0.32 \\
27 & 1140 & 1032.23 & 650.31 & 1525.00 & 1.59 & 0.36 \\
At $36{ }^{\circ} \mathrm{C}$ & & & & & & \\
9 & 2000 & 4059.12 & 1380.11 & 5359.16 & 2.94 & 0.21 \\
18 & 1200 & 1851.21 & 1166.26 & 2734.94 & 1.59 & 0.36 \\
27 & 580 & 823.17 & 617.38 & 1286.21 & 1.33 & 0.41 \\
\hline
\end{tabular}
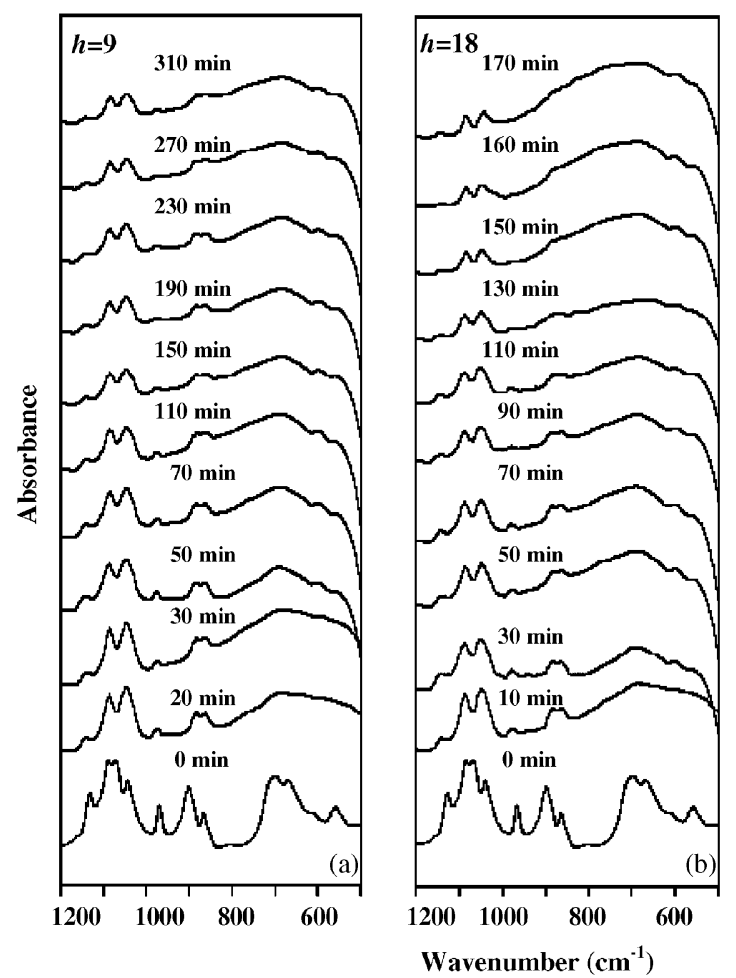

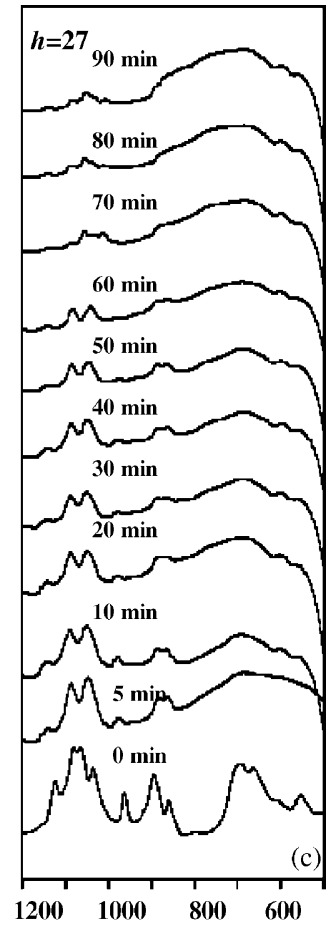

Fig. 7. FT-IR spectra of alumina gel during sol-gel process for $\mathrm{pH} 9$ at $25^{\circ} \mathrm{C}$ and various hydrolysis ratio of (a) $h=9$; (b) $h=18$; (c) $h=27$. 
Many studies of critical gel rheological properties were found that most results show $n \geq 0.5$. Values of $n$ below 0.5 have also been found [23,26-29]. Winter reported that if $n>0.5$, the gel point preceded the intersection of $G^{\prime}$ and $G^{\prime \prime}$. In contrast, if $n<0.5$, the intersection occurs before the gelation. This is coincident with the results from our system.

The value obtained in our system was very close to the one found for polycaprolactone. Izuka et al. [25] suggested that a low $\mathrm{n}$ value implies that the material at the gel point is a mostly elastic body with the limit of $G^{\prime \prime}=0$ at $n=0$ and vice versa. The low value of $n$ found for our case is probably due to the presence of physical cross-links with a finite lifetime, in addition to the permanent chemical cross-links leading to a denser network with elastic behaviour.

3.1.1.1. Effect of $p H$. The systems with the $\mathrm{pH}$ ranging from 3 to 11 at $h=9$ and temperature of $25^{\circ} \mathrm{C}$ were also studied. The values of rheological properties at the gel point were provided in Table 1 . The gelation time at each $\mathrm{pH}$ was obtained from plots of $\tan \delta$ against time in the same manner as Fig. 2. The relaxation exponent $\mathrm{n}$ was calculated from Eq. (3) at $0.8 \mathrm{rad} \mathrm{s}^{-1}$. The maximum gelation rate was observed near $\mathrm{pH} 11$, with significantly decreasing the rate at lower $\mathrm{pH}$. As investigated, the value of $t_{\mathrm{gel}}$ at higher $\mathrm{pH}$ is less than that of $t_{\mathrm{gel}}$ at lower $\mathrm{pH}$. The maximum at around $\mathrm{pH} 9$ corresponds to the isoelectric point of alumina [30]. However, it is also evident, Table 1, that the strength of the gel network formed at lower $\mathrm{pH}$ (determined from the value of $G^{\prime} / G^{\prime \prime}$ at $\left.t_{\mathrm{gel}}\right)$ is substantially higher than that obtained at higher $\mathrm{pH}$. This is consistent with a decrease of $\mathrm{n}$ values with decreasing $\mathrm{pH}$. As pointed out previously, a lower value of $\mathrm{n}$ is related to higher elastic body implying that intermolecular cross-links were stronger. This could be described that when the value of $\mathrm{pH}$ is high, molecular networks formed quickly via the reactions of hydrolysis and condensation have low interconnectivity. Consequently, the network is preferably loose with lower value of $G^{\prime}$ and higher value of $\mathrm{n}$. On the other hand, the gelation of a system with $\mathrm{pH} 9$ having no addition of catalyst can be formed slowly leading to a denser and more structured network. Therefore, the value of $G^{\prime}$ will be higher, whereas the value of $\mathrm{n}$ will be lower. Above $\mathrm{pH}$ 9 , the growth of networks occurred rapidly leading to less time to arrange itself. Thus, the obtained networks having lower value of $G^{\prime}$ and higher value of $n$ are looser. It could also be said that a gel with a longer gel time shows more elastic behavior at the gel point than the one with a shorter gel time.

3.1.1.2. Effect of hydrolysis ratio. The dynamic viscoelastic properties at the gel point were demonstrated in Table 2 for each set of reaction conditions at $25^{\circ} \mathrm{C}$. The increase of hydrolysis ratio reduced the times required for gelation. Also, $G^{\prime}$ outweighed $G^{\prime \prime}$ for all systems showing that the elastic component appreciably predominated over the viscous one at the gel point. Furthermore, $G^{\prime}$ and $\eta^{*}$ decreased with increase of hydrolysis ratio while the relaxation expo- nent increased with increase of hydrolysis ratio. It could be justified that when the hydrolysis ratio is greater, gel formation evolving quickly through the reaction of hydrolysis and condensation contains low interconnected networks. Consequently, the network is looser with lower value of $G^{\prime}$. On the other hand, a system with a lower hydrolysis ratio grew slowly resulting in a denser network with higher $G^{\prime}$. Correspondingly, a higher value of $\mathrm{n}$ is related to a lower elastic system indicating that intermolecular cross-links are weaker.

3.1.1.3. Effect of temperature. The temperature at which the measurement was performed is one of the most important factors influencing the gel time. The results for the systems varying between $20^{\circ}$ and $36^{\circ} \mathrm{C}$ were reported in Table 3 .
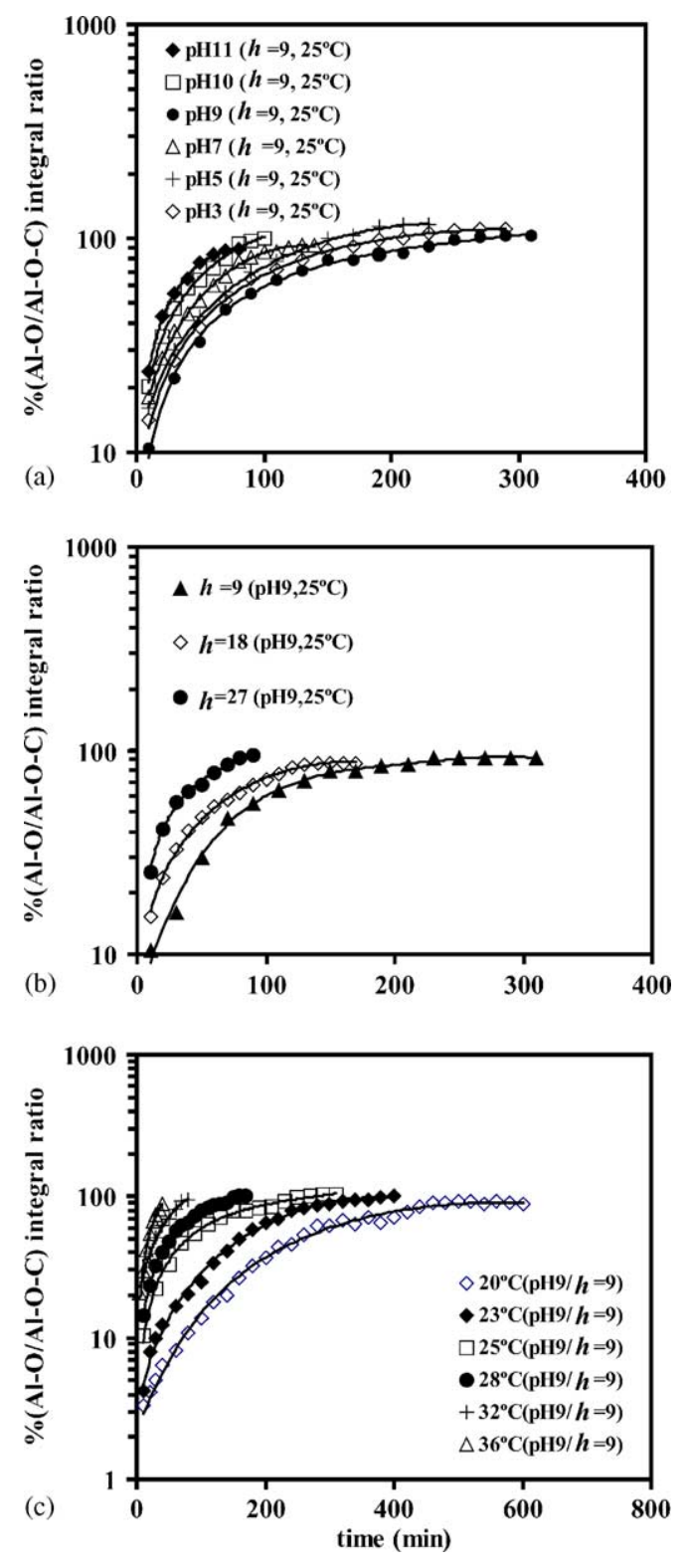

Fig. 8. Variation of hydrolysed alumatrane as a function of the reaction time at (a) $\mathrm{pH}$ dependence, (b) hydrolysis ratio dependence and (c) temperature dependence. 
At higher temperatures gelation occurs faster, resulting in a shorter time to gel. The values of the dynamic moduli at the gel point decreased with increasing temperature. The trend of these results is analogous to that of other studies [25,29]. In addition, the temperature dependence of the complex dynamic viscosity $\eta^{*}$ was also similar to that of the dynamic moduli. In contrast, the $n$ values obtained for all systems increased with temperature. These observations indicate that the network formation proceeded faster with increasing temperature, whereas the gel texture is rather loose. There are some discussions in the literature regarding an apparent activation energy for the gelation reaction calculated from the gel time at different temperatures [29,31]. Because gelation represented a specific extent of reaction, the temperature dependence of the time to gel should be described by the Arrhenius equation

$\ln \left(t_{\mathrm{gel}}\right)=A+\frac{E_{\mathrm{a}}}{R T}$

where $A$ is a constant, $R$ the ideal gas constant, and $T$ is temperature. The activation energy $E_{\mathrm{a}}$ of the gelation can be calculated from the slope of a plot of $\log t_{\mathrm{gel}}$ against $1 / T$. The Arrhenius plots for our system are shown in Fig. 6. The values of $E_{\mathrm{a}}$ were estimated to be approximately the same in each case, slightly varying around $139 \mathrm{~kJ} \mathrm{~mol}^{-1}$. This implies that $E_{\mathrm{a}}$ of the gelation does not depend on hydrolysis ratio.

\subsubsection{FT-IR analysis}

The FT-IR spectra of the gel as a function of time were compared to elucidate the study of the hydrolysed alumatrane in sol-gel process. As the reaction proceeded, a decrease in the intensity of the $\gamma(\mathrm{Al}-\mathrm{O}-\mathrm{C})$ stretching vibration of alumatrane at $1087 \mathrm{~cm}^{-1}$ was observed. Some peaks present at $0 \mathrm{~min}$. in the $900-500 \mathrm{~cm}^{-1}$ regions corresponding to $\mathrm{C}-\mathrm{H}, \mathrm{C}-\mathrm{O}$ and $\mathrm{Al}-\mathrm{O}-\mathrm{C}$ deformation vibrations disappeared, while the width and intensity of this spectral range increased with increasing the reaction time. This result corresponded to the formation of $\mathrm{Al}-\mathrm{O}-\mathrm{Al}$ bonds according to the condensation reaction. Fig. 7 depicts the FT-IR spectra of hydrolysed systems at different hydrolysis ratio. The intensity of Al-O-Al was maximised at $h=27$ with decreasing the intensity at lower hydrolysis ratio. The decrease in intensity of $\mathrm{Al}-\mathrm{O}-\mathrm{C}$ band is accompanied with the increase of $\mathrm{Al}-\mathrm{O}-\mathrm{Al}$ band as hydrolysis ratio increases. This implies that the gelation can be accelerated with increasing hydrolysis ratio leading to the reduction of the gel time.

In order to confirm the above conclusion, a careful deconvolution of the IR profiles using a computer program was also investigated. The results of the analysis are shown in the relationship between the peak ratio of $\mathrm{Al}-\mathrm{O}-\mathrm{C}$ band $\left(1087 \mathrm{~cm}^{-1}\right)$ and $\mathrm{Al}-\mathrm{O}-\mathrm{Al}$ band $\left(683 \mathrm{~cm}^{-1}\right)$ with time (Fig. 8). This is only a semiquantitative picture of the gelation of alumatrane molecule. Generally, the gel time is decreased by factors that increase the condensation rate. It is obvious that values of $\mathrm{pH}$, hydrolysis ratio and temperature are the important factors affecting the gel time. The results obtained here compared favourably with earlier study by qualitative FT-IR. Furthermore, it was found that these FT-IR observations are consistent with the rheological results.

\subsection{Characterisation of pyrolysed gel}

Some preliminary studies of pyrolysed gel were performed to examine the influence of pyrolysis temperature

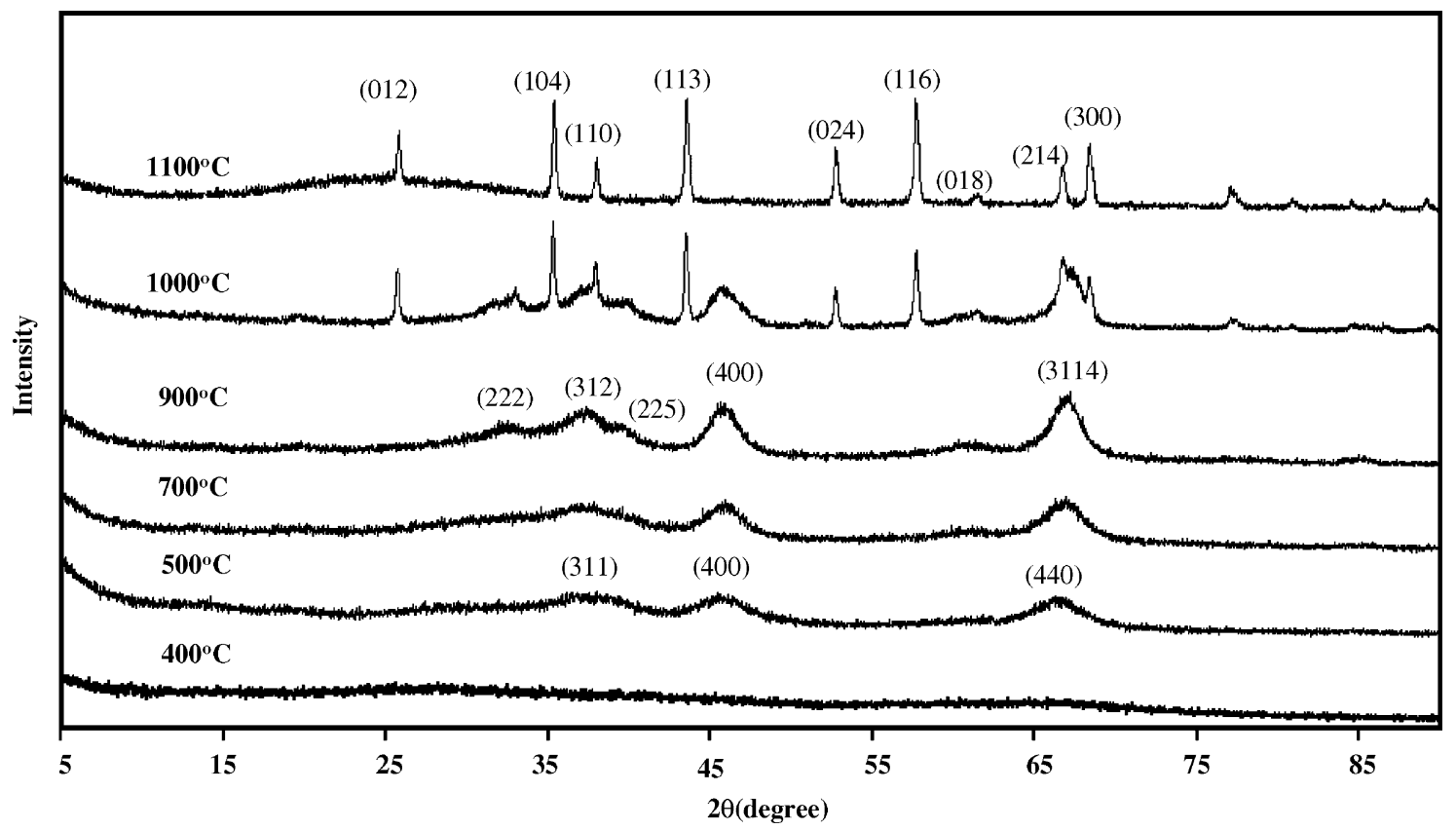

Fig. 9. XRD patterns of hydrolysed alumatrane at various pyrolysis temperatures of (a) $400{ }^{\circ} \mathrm{C}$, (b) $500{ }^{\circ} \mathrm{C},(\mathrm{c}) 700{ }^{\circ} \mathrm{C},(\mathrm{d}) 900{ }^{\circ} \mathrm{C}$, (e) $1000{ }^{\circ} \mathrm{C}$ and (f) $1100^{\circ} \mathrm{C}$. 

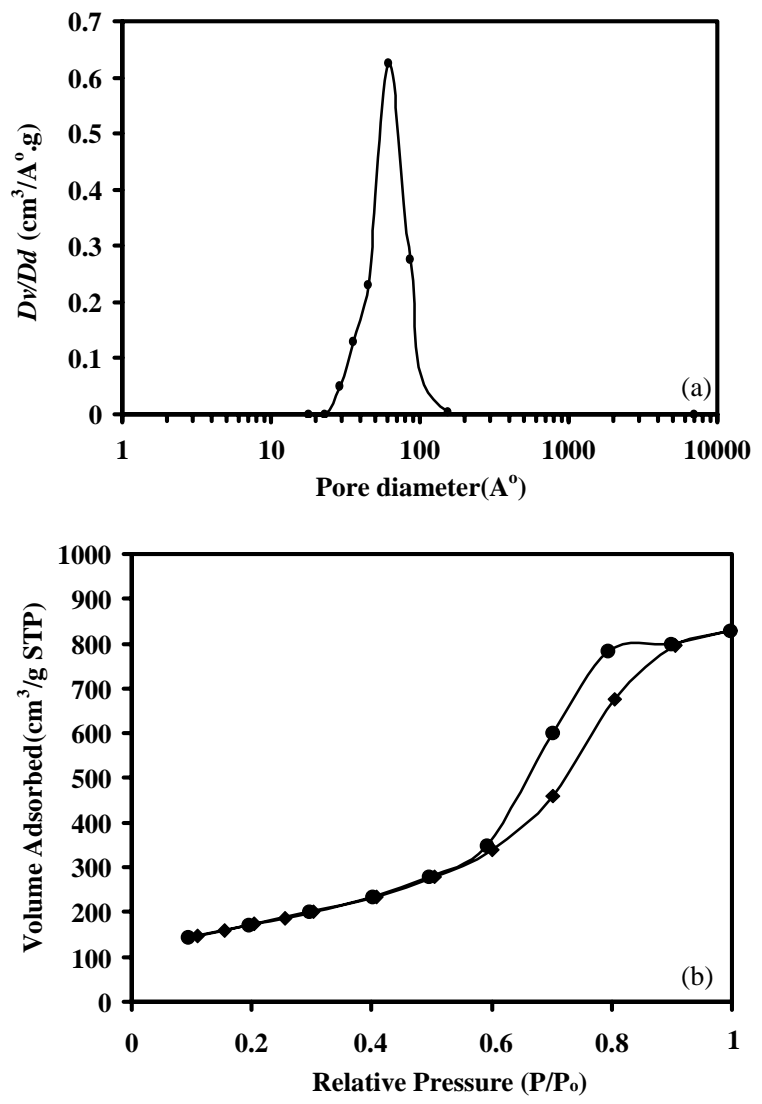

Fig. 10. Plots showing (a) Pore size distribution; (b) nitrogen adsorption-desorption isotherms of the alumatrane gel pyrolysed at $500^{\circ} \mathrm{C}$ for $7 \mathrm{~h}$.

on the properties of alumina obtained from the heat treatment of alumatrane gels. XRD patterns of pyrolysed gel at different temperatures ranging between 400 and $1100^{\circ} \mathrm{C}$ showed amorphous nature at $400{ }^{\circ} \mathrm{C}$ in Fig. 9. The sample treated at $500^{\circ} \mathrm{C}$ presents three $\gamma$-alumina peaks (ICDD, File 29-63). At $900{ }^{\circ} \mathrm{C}$, the XRD pattern of $\gamma$-alumina is better defined, however, characteristic peaks of $\delta$-alumina are not well resolved (ICDD, File 16-394). After heating at $1000{ }^{\circ} \mathrm{C}$, two phases of $\delta$-alumina and $\alpha$-alumina were detected. When the sample was heated from 1000 to $1100^{\circ} \mathrm{C}$, the $\delta$-alumina was totally transformed into $\alpha$-alumina.

The sample treated at $500{ }^{\circ} \mathrm{C}$ shows the monomodal pore size distribution in the mesopore range having a maximum centre around a pore diameter of $70 \AA$ (Fig. 10a). Also, the resulting powder shows the nitrogen adsorption-desorption isotherms of type IV (IUPAC classification) which exhibit hysteresis loops mostly of type H2 (Fig. 10b) [32]. Further investigations on the pyrolysis of alumina gel obtained various reaction conditions are in progress and will be reported later.

\section{Conclusions}

Alumatrane can be used as metal alkoxide precursor for preparing high surface alumina powders via sol-gel route.
The multiple-waveform rheological technique and FT-IR were found to be effective for studying the gelation of alumatrane. The operation variables, viz. $\mathrm{pH}$, hydrolysis ratio and temperature, affected dramatically the gel time. An increase of these parameters leads to the reduction of gel time. However, the strength of the gel network formed more quickly is lower than that obtained gradually. The value of $\mathrm{n}$ for our systems was found to be fairly low indicating high elastic gel. The apparent activation energy of the gelation reaction determined directly from gel time measurement at different temperatures was around $139 \mathrm{~kJ} \mathrm{~mol}^{-1}$. Heat treatment of the resulting alumatrane gels at $500{ }^{\circ} \mathrm{C}$ produces a homogeneous mesopore alumina having high surface area.

\section{Acknowledgements}

This work was supported by the Thailand Research Fund (TRF), Postgraduate Education and Research Program in Petroleum and Petrochemical Technology, PPT consortium (ADB) Fund and Ratchadapisake Sompote Fund, Chulalongkorn University.

\section{References}

[1] C.G. Guizard, A.C. Julbe, A. Ayral, J. Mat. Chem. 9 (1999) 55.

[2] D. Quanttrini, D. Serrano, S. Perez Catán, Granular Matter 3 (2001) 125.

[3] J. Livage, Solid State Ionics 86-88 (1996) 935.

[4] S. Suda, K. Yamashita, T. Umegaki, Solid State Ionics 89 (1996) 75 .

[5] C.A. Browne, D.H. Tarrant, M.S. Olteanu, J.W. Mullens, E.L. Chronister, Anal. Chem. 68 (1996) 2289.

[6] K. Kuraoka, H. Tanaka, T. Yazawa, J. Mater. Sci. Lett. 15 (1996) 1.

[7] T. Skapin, E. Kemnitz, Catal. Lett. 40 (1996) 241.

[8] A.A. Roger, D.K. Bruce, J. Non-Cryst. Solids 99 (1988) 359.

[9] J. Blanchard, S. Barboux-Doeuff, J. Maquet, C. Sanchez, New J. Chem. 19 (1995) 929.

[10] V. Jarayaman, T. Guanasekaran, G. Periaswami, Mater. Lett. 30 (1997) 157

[11] B.E. Yoldas, J. Non-Cryst. Solids 147 (1992) 614.

[12] H. Uchilhashi, N. Tohge, T. Minami, J. Ceram. Soc. Jpn. Int. 97 (1989) 389.

[13] B.E. Yoldas, J. Appl. Chem. Biotechnol. 23 (1973) 803.

[14] B.E. Yoldas, Ceram. Bull. 54 (1975) 286.

[15] B.E. Yoldas, Ceram. Bull. 54 (1975) 289.

[16] A.C. Pierre, D.R. Uhlmann, J. Am. Ceram. Soc. 70 (1987) 28.

[17] T. Ogihara, H. Nakajima, T. Yanagawa, N. Ogata, K. Yoshida, J. Am. Ceram. Soc. 74 (1991) 2263.

[18] Y. Opornsawad, B. Ksapabutr, S. Wongkasemjit, R.M. Laine, Eur. Polym. J. 37 (2001) 1877.

[19] M. Djabourov, J. Leblond, P. Papon, J. Phys. France 49 (1988) 333.

[20] C.M. Tung, J. Dynes, J. Appl. Polym. Sci. 27 (1982) 569.

[21] H.H. Winter, F. Chambon, J. Rheol. 31 (1987) 683.

[22] H.H. Winter, F. Chambon, J. Rheol. 30 (1986) 367.

[23] Y.G. Lin, D.T. Mallin, J.C.W. Chien, H.H. Winter, Macromolecules 24 (1991) 850.

[24] J.M. Yu, R. Jerome, P. Teyssie, Polymer 38 (1997) 347. 
[25] A. Izuka, H.H. Winter, T. Hashimoto, Macromolecules 25 (1992) 2422.

[26] A. Ponton, S. Barboux-Doeuff, C. Sanchez, Colloids Surf. A: Physicochem. Eng. Aspects 162 (1999) 177.

[27] J. Scanlan, H.H. Winter, Macromolecules 24 (1991) 47.

[28] D.F. Hodgson, E.J. Amis, Macromolecules 23 (1990) 2512.
[29] S.R. Raghavan, L.A. Chen, C. Mc Dowell, S.A. Khan, Polymer 37 (1996) 5869.

[30] G.A. Parks, Chem. Rev. 65 (1965) 177.

[31] L.J. Gough, I.T. Smith, J. Appl. Polym. Sci. 3 (1960) 362.

[32] G. Leofanti, M. Padovan, G. Tozzola, B. Venturelli, Catal. Today 41 (1998) 207. 